Korean J. Math. 21 (2013), No. 4, pp. 365-374

http://dx.doi.org/10.11568/kjm.2013.21.4.365

\title{
REMARK ON AVERAGE OF CLASS NUMBERS OF FUNCTION FIELDS
}

\author{
HWANYUP JUNG
}

\begin{abstract}
Let $k=\mathbb{F}_{q}(T)$ be a rational function field over the finite field $\mathbb{F}_{q}$, where $q$ is a power of an odd prime number, and $\mathbb{A}=\mathbb{F}_{q}[T]$. Let $\gamma$ be a generator of $\mathbb{F}_{q}^{*}$. Let $\mathcal{H}_{n}$ be the subset of $\mathbb{A}$ consisting of monic square-free polynomials of degree $n$. In this paper we obtain an asymptotic formula for the mean value of $L\left(1, \chi_{\gamma D}\right)$ and calculate the average value of the ideal class number $h_{\gamma D}$ when the average is taken over $D \in \mathcal{H}_{2 g+2}$.
\end{abstract}

\section{Introduction and statement of result}

Let $k=\mathbb{F}_{q}(T)$ be a rational function field over the finite field $\mathbb{F}_{q}$, where $q$ is a power of an odd prime number, and $\mathbb{A}=\mathbb{F}_{q}[T]$. Let $\mathbb{A}^{+}$be the set of monic polynomials in $\mathbb{A}$ and $\mathcal{H}$ be the subset of $\mathbb{A}^{+}$consisting of monic square-free polynomials. Write $\mathbb{A}_{n}^{+}=\left\{N \in \mathbb{A}^{+}: \operatorname{deg} N=n\right\}$ and $\mathcal{H}_{n}=\mathcal{H} \cap \mathbb{A}_{n}^{+}$. For any nonconstant square free $D \in \mathbb{A}^{+}$, let $\mathcal{O}_{D}$ be the integral closure of $\mathbb{A}$ in $k(\sqrt{D})$ and $h_{D}$ be the ideal class number of $\mathcal{O}_{D}$. Hoffstein and Rosen [3] calculated the average value of the ideal class number $h_{D}$ when the average is taken over all monic polynomials

Received July 3, 2013. Revised September 13, 2013. Accepted September 13, 2013.

2010 Mathematics Subject Classification: 11R58, 11R18, 11R29.

Key words and phrases: Mean Values of $L$-functions, finite fields, function fields, class numbers.

This research was supported by Basic Science Research Program through the National Research Foundation of Korea(NRF) funded by the Ministry of Education(2010-0008139).

(c) The Kangwon-Kyungki Mathematical Society, 2013.

This is an Open Access article distributed under the terms of the Creative commons Attribution Non-Commercial License (http://creativecommons.org/licenses/by -nc/3.0/) which permits unrestricted non-commercial use, distribution and reproduction in any medium, provided the original work is properly cited. 
$D$ of a fixed odd degree. Andrade [1] obtained an asymptotic formula for the mean value of $L\left(1, \chi_{D}\right)$ and calculated the average value of the ideal class number $h_{D}$ when the average is taken over $D \in \mathcal{H}_{2 g+1}$. We remark that Andrade assumed that $q \equiv 1 \bmod 4$ for simplicity, but his results hold true for any odd $q>3$. In a recent paper, the author [4] obtained an asymptotic formula for the mean value of $L\left(1, \chi_{D}\right)$ and calculated the average value of the ideal class number $h_{D}$ when the average is taken over $D \in \mathcal{H}_{2 g+2}$. Note that if $D \in \mathcal{H}_{2 g+1}$, the infinite place $\infty_{k}=(1 / T)$ of $k$ ramifies in $k(\sqrt{D})$, i.e., $k(\sqrt{D}) / k$ is a (ramified) imaginary quadratic extension, and if $D \in \mathcal{H}_{2 g+2}, \infty_{k}$ splits in $k(\sqrt{D})$, i.e., $k(\sqrt{D}) / k$ is a real quadratic extension. Let $\gamma$ be a fixed generator of $\mathbb{F}_{q}^{*}$. Any inert imaginary quadratic extension $K$ of $k$ (i.e., $\infty_{k}$ is inert in $K$ ) can be written uniquely in the form $K=k(\sqrt{\gamma D})$ for some $D \in \mathcal{H}_{2 g+2}$. The aim of this paper is to study the asymptotic formula for the mean value of $L\left(1, \chi_{\gamma D}\right)$ and calculate the average value of the ideal class number $h_{\gamma D}$ when the average is taken over $D \in \mathcal{H}_{2 g+2}$. We state our main results.

Theorem 1.1. We have

$$
\sum_{D \in \mathcal{H}_{2 g+2}} L\left(1, \chi_{\gamma D}\right)=|D| \mathrm{P}(2)+O\left(2^{g} q^{g}\right)
$$

where $|D|=q^{2 g+2}$ and

$$
\mathrm{P}(s)=\prod_{\substack{P \in \mathbb{A}^{+} \\ \text {irreducible }}}\left(1-\frac{1}{(1+|P|)|P|^{s}}\right) .
$$

Since $\sharp \mathcal{H}_{2 g+2}=(q-1) q^{2 g+1}$ (see $\left.(2.1)\right)$, as a corollary of the Theorem 1.1, we have the following.

Corollary 1.2. We have

as $g \rightarrow \infty$.

$$
\frac{1}{\sharp \mathcal{H}_{2 g+2}} \sum_{D \in \mathcal{H}_{2 g+2}} L\left(1, \chi_{\gamma D}\right) \sim|D| \mathrm{P}(2)
$$

For any $D \in \mathcal{H}_{2 g+2}$, we have the following class number formula (see [3, Theorem 0.6]):

$$
L\left(1, \chi_{\gamma D}\right)=\frac{q+1}{2 \sqrt{|D|}} h_{\gamma D}=\frac{q \zeta_{\mathbb{A}}(2)}{2 \zeta_{\mathbb{A}}(3) \sqrt{|D|}} h_{\gamma D} .
$$


By Corollary 1.2 and the class number formula (1.1), we have the following asymptotic formula for the average of the class number $h_{\gamma D}$.

Theorem 1.3. We have

$$
\frac{1}{\sharp \mathcal{H}_{2 g+2}} \sum_{D \in \mathcal{H}_{2 g+2}} h_{\gamma D} \sim \frac{2 \zeta_{\mathbb{A}}(3) \mathrm{P}(2)}{q \zeta_{\mathbb{A}}(2)}|D| \sqrt{|D|}
$$

as $g \rightarrow \infty$.

\section{Preliminaries}

2.1. Quadratic Dirichlet $L$-function. Let $\mathbb{A}^{+}$be the set of all monic polynomials in $\mathbb{A}$ and $\mathbb{A}_{n}^{+}=\left\{N \in \mathbb{A}^{+}: \operatorname{deg} N=n\right\}(n \geq 0)$. The zeta function $\zeta_{\mathbb{A}}(s)$ of $\mathbb{A}$ is defined by the infinite series

$$
\zeta_{\mathbb{A}}(s)=\sum_{N \in \mathbb{A}^{+}}|N|^{-s} .
$$

It is straightforward to see that $\zeta_{\mathbb{A}}(s)=\frac{1}{1-q^{1-s}}$. For any square-free $D \in \mathbb{A}$, the quadratic character $\chi_{D}$ is defined by the Jacobi symbol $\chi_{D}(N)=\left(\frac{D}{N}\right)$ and the quadratic Dirichlet $L$-function $L\left(s, \chi_{D}\right)$ associated to $\chi_{D}$ is

$$
L\left(s, \chi_{D}\right)=\sum_{N \in \mathbb{A}^{+}} \chi_{D}(N)|N|^{-s} .
$$

We can write $L\left(s, \chi_{D}\right)=\sum_{n=0}^{\infty} \sigma_{n}(D) q^{-n s}$ with $\sigma_{n}(D)=\sum_{N \in \mathbb{A}_{n}^{+}} \chi_{D}(N)$. Since $\sigma_{n}(D)=0$ for $n \geq \operatorname{deg} D, L\left(s, \chi_{D}\right)$ is a polynomial in $q^{-s}$ of degree $\leq \operatorname{deg} D-1$. Putting $u=q^{-s}$, write

$$
\mathcal{L}\left(u, \chi_{D}\right)=\sum_{n=0}^{\operatorname{deg} D-1} \sigma_{n}(D) u^{n}=L\left(s, \chi_{D}\right) .
$$

The cardinality of $\mathcal{H}_{n}$ is $\# \mathcal{H}_{1}=q$ and $\# \mathcal{H}_{n}=\left(1-q^{-1}\right) q^{d}(n \geq 2)$. In particular, we have

$$
\# \mathcal{H}_{2 g+2}=(q-1) q^{2 g+1}=\frac{q^{2 g+2}}{\zeta_{\AA}(2)} .
$$

Fix a generator $\gamma$ of $\mathbb{F}_{q}^{*}$. Write $\bar{D}=\gamma D$ for any $D \in H_{2 g+2}$. Since $\left(\frac{\gamma}{N}\right)=$ $(-1)^{\operatorname{deg} N}$, we have $\left(\frac{\bar{D}}{N}\right)=(-1)^{\operatorname{deg} N}\left(\frac{D}{N}\right)$. Hence, $\sigma_{n}(\bar{D})=(-1)^{n} \sigma_{n}(D)$. 
For $D \in \mathcal{H}_{2 g+2}, \mathcal{L}\left(u, \chi_{\bar{D}}\right)$ has a trivial zero at $u=-1$. The complete $L$-function $\tilde{\mathcal{L}}\left(u, \chi_{\bar{D}}\right)$ is defined by

$$
\tilde{\mathcal{L}}\left(u, \chi_{\bar{D}}\right)=(1+u)^{-1} \mathcal{L}\left(u, \chi_{\bar{D}}\right) .
$$

It is a polynomial of even degree $2 g$ and satisfies the functional equation

$$
\tilde{\mathcal{L}}\left(u, \chi_{\bar{D}}\right)=\left(q u^{2}\right)^{g} \tilde{\mathcal{L}}\left((q u)^{-1}, \chi_{\bar{D}}\right)
$$

Lemma 2.1. Let $\chi_{\bar{D}}$ be a quadratic character, where $D \in \mathcal{H}_{2 g+2}$. Then

$$
\begin{aligned}
\mathcal{L}\left(q^{-1}, \chi_{\bar{D}}\right)= & \sum_{n=0}^{g}(-1)^{n} q^{-n} \sum_{N \in \mathbb{A}_{n}^{+}} \chi_{D}(N)+(-1)^{g} q^{-(g+1)} \sum_{n=0}^{g} \sum_{N \in \mathbb{A}_{n}^{+}} \chi_{D}(N) \\
& +\left(1+q^{-1}\right) q^{-g} \sum_{n=0}^{g-1}\left(\frac{(-1)^{n}+(-1)^{g+1}}{2}\right) \sum_{N \in \mathbb{A}_{n}^{+}} \chi_{D}(N) .
\end{aligned}
$$

Proof. Write $\tilde{\mathcal{L}}\left(u, \chi_{\bar{D}}\right)=\sum_{n=0}^{2 g} \tilde{\sigma}_{n}(\bar{D}) u^{n}$. Since $\mathcal{L}\left(u, \chi_{\bar{D}}\right)=(1+$ $u) \tilde{\mathcal{L}}\left(u, \chi_{\bar{D}}\right)$, we have $\sigma_{0}(\bar{D})=\tilde{\sigma}_{0}(\bar{D}), \sigma_{n}(\bar{D})=\tilde{\sigma}_{n-1}(\bar{D})+\tilde{\sigma}_{n}(\bar{D})(1 \leq$ $n \leq 2 g)$ and $\sigma_{2 g+1}(\bar{D})=\tilde{\sigma}_{2 g}(\bar{D})$, or

$$
\tilde{\sigma}_{n}(\bar{D})=\sum_{i=0}^{n}(-1)^{n-i} \sigma_{i}(\bar{D})(0 \leq n \leq 2 g) .
$$

By substituting $\tilde{\mathcal{L}}\left(u, \chi_{\bar{D}}\right)=\sum_{n=0}^{2 g} \tilde{\sigma}_{n}(\bar{D}) u^{n}$ into $(2.2)$ and equating coefficients, we have $\tilde{\sigma}_{n}(\bar{D})=\tilde{\sigma}_{2 g-n}(\bar{D}) q^{-g+n}$ or $\tilde{\sigma}_{2 g-n}(\bar{D})=\tilde{\sigma}_{n}(\bar{D}) q^{g-n}$. Hence,

$$
\tilde{\mathcal{L}}\left(u, \chi_{\bar{D}}\right)=\sum_{n=0}^{g} \tilde{\sigma}_{n}(\bar{D}) u^{n}+q^{g} u^{2 g} \sum_{n=0}^{g-1} \tilde{\sigma}_{n}(\bar{D}) q^{-n} u^{-n}
$$

In particular, we have

$$
\tilde{\mathcal{L}}\left(q^{-1}, \chi_{\bar{D}}\right)=\sum_{n=0}^{g} \tilde{\sigma}_{n}(\bar{D}) q^{-n}+q^{-g} \sum_{n=0}^{g-1} \tilde{\sigma}_{n}(\bar{D}) .
$$


By substituting (2.3) into (2.4) and using $\sigma_{n}(\bar{D})=(-1)^{n} \sigma_{n}(D)$, we have

$$
\begin{aligned}
\tilde{\mathcal{L}}\left(q^{-1}, \chi_{\bar{D}}\right)= & \frac{1}{1+q^{-1}} \sum_{n=0}^{g}(-1)^{n} q^{-n} \sigma_{n}(D)+\frac{(-1)^{g} q^{-(g+1)}}{1+q^{-1}} \sum_{n=0}^{g} \sigma_{n}(D) \\
& +q^{-g} \sum_{n=0}^{g-1}\left(\frac{(-1)^{n}+(-1)^{g+1}}{2}\right) \sigma_{n}(D) .
\end{aligned}
$$

So we get the result since $\mathcal{L}\left(q^{-1}, \chi_{\bar{D}}\right)=\left(1+q^{-1}\right) \tilde{\mathcal{L}}\left(q^{-1}, \chi_{\bar{D}}\right)$.

2.2. Contribution of square parts. The square part contributions in the summation of $L\left(1, \chi_{\bar{D}}\right)$ over $D \in \mathcal{H}_{2 g+2}$ are given as follow.

Proposition 2.2. We have

$$
\sum_{m=0}^{\left[\frac{g}{2}\right]} q^{-2 m} \sum_{L \in \mathbb{A}_{m}^{+}} \sum_{\substack{D \in \mathcal{H}_{2 g+2} \\(L, D)=1}} 1=|D| \mathrm{P}(2)-q^{-\left(\left[\frac{g}{2}\right]+1\right)}|D| \mathrm{P}(1)+O\left(q^{g}\right)
$$

$$
(-1)^{g} q^{-(g+1)} \sum_{m=0}^{\left[\frac{g}{2}\right]} \sum_{L \in \mathbb{A}_{m}^{+}} \sum_{\substack{D \in \mathcal{H}_{2 g+2} \\(L, D)=1}} 1=(-1)^{g} q^{-(g+1)+\left[\frac{g}{2}\right]}|D| \mathrm{P}(1)+O\left(g q^{g}\right)
$$

and

$$
\begin{aligned}
\left(1+q^{-1}\right) q^{-g}\left(\frac{1+(-1)^{g+1}}{2}\right) \sum_{m=0}^{\left[\frac{g-1}{2}\right]} \sum_{L \in \mathbb{A}_{m}^{+}} \sum_{\substack{D \in \mathcal{H}_{2 g+2} \\
(L, D)=1}} 1 \\
=(q+1) q^{-g+\left[\frac{g-1}{2}\right]-1}\left(\frac{1+(-1)^{g+1}}{2}\right)|D| \mathrm{P}(1)+O\left(g q^{g}\right) .
\end{aligned}
$$

Proof. The proofs are mild modifications of those of Proposition 3.7 in [4]. We only give the proof of (2.7). By using the fact that (see [2, Proposition 5.2]))

$$
\sum_{\substack{D \in \mathcal{H}_{2 g+2} \\(D, L)=1}} 1=\frac{|D|}{\zeta_{\mathbb{A}}(2)} \prod_{P \mid L}\left(1+|P|^{-1}\right)^{-1}+O\left(\sqrt{|D|} \frac{\Phi(L)}{|L|}\right)
$$


we have

$$
\begin{aligned}
\left(1+q^{-1}\right) q^{-g}\left(\frac{1+(-1)^{g+1}}{2}\right) \sum_{m=0}^{\left[\frac{g-1}{2}\right]} \sum_{L \in \mathbb{A}_{m}^{+}} \sum_{\substack{D \in \mathcal{H}_{2 g+2} \\
(L, D)=1}} 1 \\
=\left(1+q^{-1}\right) q^{-g}\left(\frac{1+(-1)^{g+1}}{2}\right) \frac{|D|}{\zeta_{\mathbb{A}}(2)} \sum_{m=0}^{\left[\frac{g-1}{2}\right]} \sum_{L \in \mathbb{A}_{m}^{+}} \prod_{P \mid L}\left(1+|P|^{-1}\right)^{-1} \\
+O\left(q^{-g} \sum_{m=0}^{\left[\frac{g-1}{2}\right]} \sum_{L \in \mathbb{A}_{m}^{+}} \sqrt{|D|} \frac{\Phi(L)}{|L|}\right)
\end{aligned}
$$

where $\Phi(L)$ is the Euler totient function. Using the fact that $\sum_{L \in \mathbb{A}_{m}^{+}} \Phi(L)$ $=\left(1-q^{-1}\right) q^{2 m}$ (see [5, Proposition 2.7]), we have

$$
\begin{aligned}
q^{-g} \sum_{m=0}^{\left[\frac{g-1}{2}\right]} \sum_{L \in \mathbb{A}_{m}^{+}} \sqrt{|D|} \frac{\Phi(L)}{|L|} & =q \sum_{m=0}^{\left[\frac{g-1}{2}\right]} q^{-m} \sum_{L \in \mathbb{A}_{m}^{+}} \Phi(L) \\
& =(q-1) \sum_{m=0}^{\left[\frac{g-1}{2}\right]} q^{m} \ll q^{\frac{g+1}{2}} .
\end{aligned}
$$

By using the fact that ( $[2$, Lemma 5.7])

$$
\sum_{L \in \mathbb{A}_{m}^{+}} \prod_{P \mid L}\left(1+|P|^{-1}\right)^{-1}=q^{m} \sum_{\substack{M \in \mathbb{A}^{+} \\ \operatorname{deg} M \leq m}} \frac{\mu(M)}{|M|} \prod_{P \mid M} \frac{1}{1+|P|}
$$


we have

$$
\begin{aligned}
\left(1+q^{-1}\right) q^{-g}\left(\frac{1+(-1)^{g+1}}{2}\right) \frac{|D|}{\zeta_{\mathbb{A}}(2)} \sum_{m=0}^{\left[\frac{g-1}{2}\right]} \sum_{L \in \mathbb{A}_{m}^{+}} \prod_{P \mid L}\left(1+|P|^{-1}\right)^{-1} \\
=\left(1+q^{-1}\right) q^{-g}\left(\frac{1+(-1)^{g+1}}{2}\right) \frac{|D|}{\zeta_{\mathbb{A}}(2)} \sum_{m=0}^{\left[\frac{g-1}{2}\right]} q^{m} \\
\qquad \sum_{\substack{M \in \mathbb{A}^{+} \\
\operatorname{deg} M \leq m}} \frac{\mu(M)}{|M|} \prod_{P \mid M} \frac{1}{1+|P|} \\
=(q+1) q^{-g+\left[\frac{g-1}{2}\right]-1}\left(\frac{1+(-1)^{g+1}}{2}\right)|D| \sum_{\substack{M \in \mathbb{A}^{+} \\
\operatorname{deg} M \leq\left[\frac{g-1}{2}\right]}} \frac{\mu(M)}{|M|} \prod_{P \mid M} \frac{1}{1+|P|} \\
-(q+1) q^{-(g+2)}\left(\frac{1+(-1)^{g+1}}{2}\right)|D| \sum_{\substack{M \in \mathbb{A}^{+} \\
\operatorname{deg} M \leq\left[\frac{g-1}{2}\right]}} \mu(M) \prod_{P \mid M} \frac{1}{1+|P|} .
\end{aligned}
$$

Finally, by using ( [4, Lemma 3.3, Lemma 3.5])

$$
\sum_{\substack{M \in \mathbb{A}^{+} \\ \operatorname{deg} M \leq\left[\frac{g-1}{2}\right]}} \frac{\mu(M)}{|M|} \prod_{P \mid M} \frac{1}{1+|P|}=\mathrm{P}(1)+O\left(q^{-\frac{g-1}{2}}\right)
$$

and

$$
\sum_{\substack{M \in \mathbb{A}^{+} \\ \operatorname{deg} M \leq\left[\frac{g-1}{2}\right]}} \mu(M) \prod_{P \mid M} \frac{1}{1+|P|} \leq \frac{g+1}{2}
$$

we have

$$
\begin{array}{r}
\left(1+q^{-1}\right) q^{-g}\left(\frac{1+(-1)^{g+1}}{2}\right) \frac{|D|}{\zeta_{\mathbb{A}}(2)} \sum_{m=0}^{\left[\frac{g-1}{2}\right]} \sum_{L \in \mathbb{A}_{m}^{+}} \prod_{P \mid L}\left(1+|P|^{-1}\right)^{-1} \\
=(q+1) q^{-g+\left[\frac{g-1}{2}\right]-1}\left(\frac{1+(-1)^{g+1}}{2}\right)|D| \mathrm{P}(1)+O\left(g q^{g}\right) .
\end{array}
$$


2.3. Contribution of non square parts. The non square part contributions in the summation of $L\left(1, \chi_{\bar{D}}\right)$ over $D \in \mathcal{H}_{2 g+2}$ are given as follow.

Proposition 2.3. We have

$$
\begin{aligned}
& \sum_{D \in \mathcal{H}_{2 g+2}} \sum_{n=0}^{g}(-1)^{n} q^{-n} \sum_{\substack{N \in \mathbb{A}_{n}^{+} \\
N \neq \square}} \chi_{D}(N)=O\left(2^{g} q^{g}\right), \\
& (-1)^{g} q^{-(g+1)} \sum_{D \in \mathcal{H}_{2 g+2}} \sum_{n=0}^{g} \sum_{\substack{N \in \mathbb{A}_{n}^{+} \\
N \neq \square}} \chi_{D}(N)=O\left(2^{g} q^{g}\right)
\end{aligned}
$$

and

$$
\left(1+q^{-1}\right) q^{-g} \sum_{D \in \mathcal{H}_{2 g+2}} \sum_{n=0}^{g-1}\left(\frac{(-1)^{n}+(-1)^{g+1}}{2}\right) \sum_{\substack{N \in \mathbb{A}_{n}^{+} \\ N \neq \square}} \chi_{D}(N)=O\left(2^{g} q^{g}\right) .
$$

Proof. As in [2, Lemma 6.4], for any non-square $N \in \mathbb{A}^{+}$, we have

$$
\sum_{D \in \mathcal{H}_{2 g+2}} \chi_{D}(N) \ll q^{g+1} 2^{\operatorname{deg} N-1} .
$$

Using (2.12), we have

$$
\begin{aligned}
\sum_{D \in \mathcal{H}_{2 g+2}} \sum_{n=0}^{g}(-1)^{n} q^{-n} \sum_{\substack{N \in \mathbb{A}_{n}^{+} \\
N \neq \square}} \chi_{D}(N) & \ll \sum_{n=0}^{g} q^{-n} \sum_{N \in \mathbb{A}_{n}^{+}} q^{g+1} 2^{n-1} \\
& \ll q^{g} \sum_{n=0}^{g} 2^{n} \ll 2^{g} q^{g}, \\
(-1)^{g} q^{-(g+1)} \sum_{D \in \mathcal{H}_{2 g+2}} \sum_{n=0}^{g} \sum_{\substack{N \in \mathbb{A}_{n}^{+} \\
N \neq \square}} \chi_{D}(N) & \ll q^{-(g+1)} \sum_{n=0}^{g} \sum_{N \in \mathbb{A}_{n}^{+}} q^{g+1} 2^{n-1} \\
& \ll \sum_{n=0}^{g} 2^{n} q^{n} \ll 2^{g} q^{g}
\end{aligned}
$$


and

$$
\begin{aligned}
& \left(1+q^{-1}\right) q^{-g} \sum_{D \in \mathcal{H}_{2 g+2}} \sum_{n=0}^{g-1}\left(\frac{(-1)^{n}+(-1)^{g+1}}{2}\right) \sum_{\substack{N \in \mathbb{A}_{n}^{+} \\
N \neq \square}} \chi_{D}(N) \\
& \ll\left(1+q^{-1}\right) q^{-g} \sum_{n=0}^{g-1} \sum_{N \in \mathbb{A}_{n}^{+}} q^{g+1} 2^{n-1} \\
& \ll(q+1) \sum_{n=0}^{g-1} 2^{n} q^{n} \ll 2^{g} q^{g} .
\end{aligned}
$$

\section{Proof of Theorem 1.1}

By Lemma 2.1, we have

$$
\begin{aligned}
\sum_{D \in \mathcal{H}_{2 g+2}} L\left(1, \chi_{\bar{D}}\right)= & \sum_{D \in \mathcal{H}_{2 g+2}} \sum_{n=0}^{g}(-1)^{n} q^{-n} \sum_{N \in \mathbb{A}_{n}^{+}} \chi_{D}(N)+(-1)^{g} q^{-(g+1)} \\
& \sum_{D \in \mathcal{H}_{2 g+2}} \sum_{n=0}^{g} \sum_{N \in \mathbb{A}_{n}^{+}} \chi_{D}(N) \\
+\left(1+q^{-1}\right) q^{-g} & \sum_{D \in \mathcal{H}_{2 g+2}} \sum_{n=0}^{g-1}\left(\frac{(-1)^{n}+(-1)^{g+1}}{2}\right) \sum_{N \in \mathbb{A}_{n}^{+}} \chi_{D}(N) .
\end{aligned}
$$

By (2.5) and (2.9), we have

$$
\begin{aligned}
& \sum_{D \in \mathcal{H}_{2 g+2}} \sum_{n=0}^{g}(-1)^{n} q^{-n} \sum_{N \in \mathbb{A}_{n}^{+}} \chi_{D}(N) \\
& =|D| \mathrm{P}(2)-q^{-\left(\left[\frac{g}{2}\right]+1\right)}|D| \mathrm{P}(1)+O\left(2^{g+1} q^{g+1}\right)
\end{aligned}
$$


and, by (2.6) and (2.10), we have

$$
\begin{aligned}
&(-1)^{g} q^{-(g+1)} \sum_{D \in \mathcal{H}_{2 g+2}} \sum_{n=0}^{g} \sum_{N \in \mathbb{A}_{n}^{+}} \chi_{D}(N) \\
&=(-1)^{g} q^{-(g+1)+\left[\frac{g}{2}\right]}|D| \mathrm{P}(1)+O\left(2^{g+1} q^{g+1}\right) .
\end{aligned}
$$

Similarly, by (2.7) and (2.11), we have

$$
\begin{aligned}
\left(1+q^{-1}\right) q^{-g} \sum_{D \in \mathcal{H}_{2 g+2}} \sum_{n=0}^{g-1}\left(\frac{(-1)^{n}+(-1)^{g+1}}{2}\right) \sum_{N \in \mathbb{A}_{n}^{+}} \chi_{D}(N) \\
=(q+1) q^{-g+\left[\frac{g-1}{2}\right]-1}\left(\frac{1+(-1)^{g+1}}{2}\right)|D| \mathrm{P}(1)+O\left(2^{g} q^{g}\right) .
\end{aligned}
$$

It is easy to see that

$$
(-1)^{g} q^{-(g+1)+\left[\frac{g}{2}\right]}+(q+1) q^{-g+\left[\frac{g-1}{2}\right]-1}\left(\frac{1+(-1)^{g+1}}{2}\right)-q^{-\left(\left[\frac{g}{2}\right]+1\right)}=0 .
$$

Hence, by inserting (3.2), (3.3) and (3.4) into (3.1), we get

$$
\sum_{D \in \mathcal{H}_{2 g+2}} L\left(1, \chi_{\bar{D}}\right)=|D| \mathrm{P}(2)+O\left(2^{g} q^{g}\right) .
$$

\section{References}

[1] J.C. Andrade, A note on the mean value of L-functions in function fields. Int. J. Number Theory, 08 (2012), no. 12, 1725-1740.

[2] J.C. Andrade and J.P. Keating, The mean value of $L\left(\frac{1}{2}, \chi\right)$ in the hyperelliptic ensemble. J. Number Theory 132 (2012), no. 12, 2793-2816.

[3] J. Hoffstein and M. Rosen, Average values of L-series in function fields. J. Reine Angew. Math. 426 (1992), 117-150.

[4] H. Jung, A note on the mean value of $L(1, \chi)$ in the hyperelliptic ensemble. To appear in Int. J. Number Theory.

[5] M. Rosen, Number theory in function fields. Graduate Texts in Mathematics 210, Springer-Verlag, New York, 2002.

Department of Mathematics Education

Chungbuk National University

Cheongju 361-763, Korea

E-mail: hyjung@chungbuk.ac.kr 